\title{
31. PALEOMAGNETIC RESULTS FROM DEEP SEA DRILLING PROJECT LEG 78A ${ }^{1}$
}

\author{
Douglas S. Wilson, Geophysics Department, Stanford University²
}

\begin{abstract}
The results of paleomagnetic studies of samples from DSDP Leg 78A are reported. For Site 541, the interval from 60 to $200 \mathrm{~m}$ sub-bottom was correlated with the Matuyama through Gilbert polarity epochs. For Site 543 , the interval from 150 to $190 \mathrm{~m}$ sub-bottom was correlated with marine magnetic Anomalies 5C through 5E. Down-dip directions of tilted beds inferred from declination values for Sites 541 and 542 suggest a pattern of monoclinal folding. Results from basalt samples are comparable to those from other DSDP sites in relatively old basalts.
\end{abstract}

\section{INTRODUCTION}

The primary objective of Deep Sea Drilling Project Leg 78A was to study the accretionary processes of the Barbados Ridge, at the convergent margin between the American and Caribbean plates. Site locations are shown in Figure 1. Sites 541 and 542 are near the toe of the accretionary prism and about a kilometer apart; coring at both recovered only sediment and failed to penetrate the interplate thrust fault. Site 543 was chosen as a reference east (seaward) of the deformation front, on presumably normal, undeformed ocean crust; drilling there bottomed in basalt overlain by and interbedded with Upper Cretaceous (Campanian) sediments. Because of time constraints, rotary coring was used throughout the cruise.

Paleomagnetic sampling on Leg 78A was carried out with three objectives in mind: to help determine age by polarity stratigraphy; to constrain in situ structure with estimates of bed strikes; and to characterize the magnetic properties of the basaltic basement, thereby supplementing data from other DSDP legs on the general nature of the source layer for marine magnetic anomalies. Recovery at all the sites was fairly high, but practical considerations limited the number of sediment samples suitable for paleomagnetic work. To avoid problems with drilling disturbance or rotation resulting from undetected tectonic tilting, samples were taken only at visible beds. The drilling disturbance was sufficiently severe in the upper $50 \mathrm{~m}$ or so of each hole that no samples were taken in the upper parts of any of the holes. At best, the sample interval was 1-2 m, with occasional gaps of several meters or more owing to lack of recovery or the disturbed nature of the core. In Hole 541, because of the exceptionally high sediment accumulation rate, this rather sparse sample interval was adequate for polarity stratigraphy over much of the hole.

\section{METHODS}

In the less consolidated sediments of the core, samples were taken by pressing $2.4 \mathrm{~cm} \times 2.4 \mathrm{~cm} \times 2 \mathrm{~cm}$ plastic cubes into the core. The cubes, scribed with arrows pointing upcore, were pried from the rest

${ }^{1}$ Biju-Duval, B., Moore, J. C., et al., Init. Repts. DSDP, Vol. 78A: Washington (U.S. Govt, Printing Office).

2 Address: Geophysics Department, Stanford University, Stanford, CA 94305. of the core. In the more consolidated regions, an upcore arrow was scribed directly on the core, and a sample of measurable size was sawn or drilled. The basalt samples were standard $2.5 \mathrm{~cm} \times 2.5 \mathrm{~cm}$ cores. The samples were measured on the shipboard Digico fluxgate spinner magnetometer; alternating-field (AF) demagnetization studies were carried out using the Schonstedt AF demagnetizer. For some of the specimens, follow-up studies were done at Stanford University on the Superconducting Technology two-axis cryogenic magnetometer with built-in AF demagnetizer. For the shipboard measurements, the most straightforward procedure was to imagine a hypothetical arrow on the top (upcore side) of each specimen pointing to the actual upcore arrow scribed on the side of the sample. The hypothetical arrow corresponds to the fiducial line referred to in the Digico manual, and allows the samples to be measured without using the time-consuming field or bedding corrections. The Digico convention defines the coordinate system for relative declinations: the direction normal to and away from the cut surface of the working half of the core is $0^{\circ}$, increasing clockwise looking downcore. In this coordinate system, the working half of the core is the "south" half. This same coordinate system was used to record strikes and dips of bedding for structural analysis. At each measurable bed, the core was sliced to give apparent dips in two different planes, defining the bedding plane. The strike of the bed was taken to be the line in the bedding plane normal to the axis of the core. By convention, the strikes recorded are $90^{\circ}$ counterclockwise from the down-dip direction. The magnetization directions are corrected for bedding by rotating the direction about the strike line by the amount of the dip.

The measurement results are tabulated in Table 1, which lists, for each sample, natural remanent magnetization (NRM) (in $\mathrm{A} / \mathrm{m}$ ), magnetization at optimum demagnetization level $(\mathrm{A} / \mathrm{m})$, optimum demagnetization level $(\mathrm{mT})$, polarity interpretation (normal or reversed), and reliability estimate. For samples taken at dipping beds, a second line gives strike, dip, and corrected inclinations and declinations. For the basalt samples, susceptibility (cgs) is listed instead of polarity and reliability. The reliability categories are modeled after U.S. academic course grades: A represents a stable magnetization inferred from at least two demagnetizations; $\mathrm{B}$ indicates that stable magnetization was inferred from the agreement between a single demagnetization and the NRM; $\mathrm{C}$ indicates that the magnetization direction was still changing systematically at the highest demagnetization used; D indicates a marginally stable sample; and $\mathrm{F}$ indicates an unstable one. The $\mathrm{H}$ designation indicates that the magnetization was too close to horizontal to determine the polarity. the latitude for the sites is $15^{\circ} \mathrm{N}$, which should be valid for the entire Neogene. This latitude predicts a dipole inclination of $28^{\circ}$, which is far enough from horizontal for polarity interpretation, but shallow enough that some horizontal magnetizations should be expected either from normal secular variation or from an increase in scatter caused by rotary coring. On this basis, a sample with a stable inclination greater than $+10^{\circ}$ was judged to be normal and less than $-10^{\circ}$ was considered reversed. Inclinations between $+10^{\circ}$ and $-10^{\circ}$ were not used for polarity determinations. The expected inclination for older times is not clear, and depends on whether the sites have been on the North American Plate or South American Plate. (The active boundary between these two plates has never been clearly recognized.) 


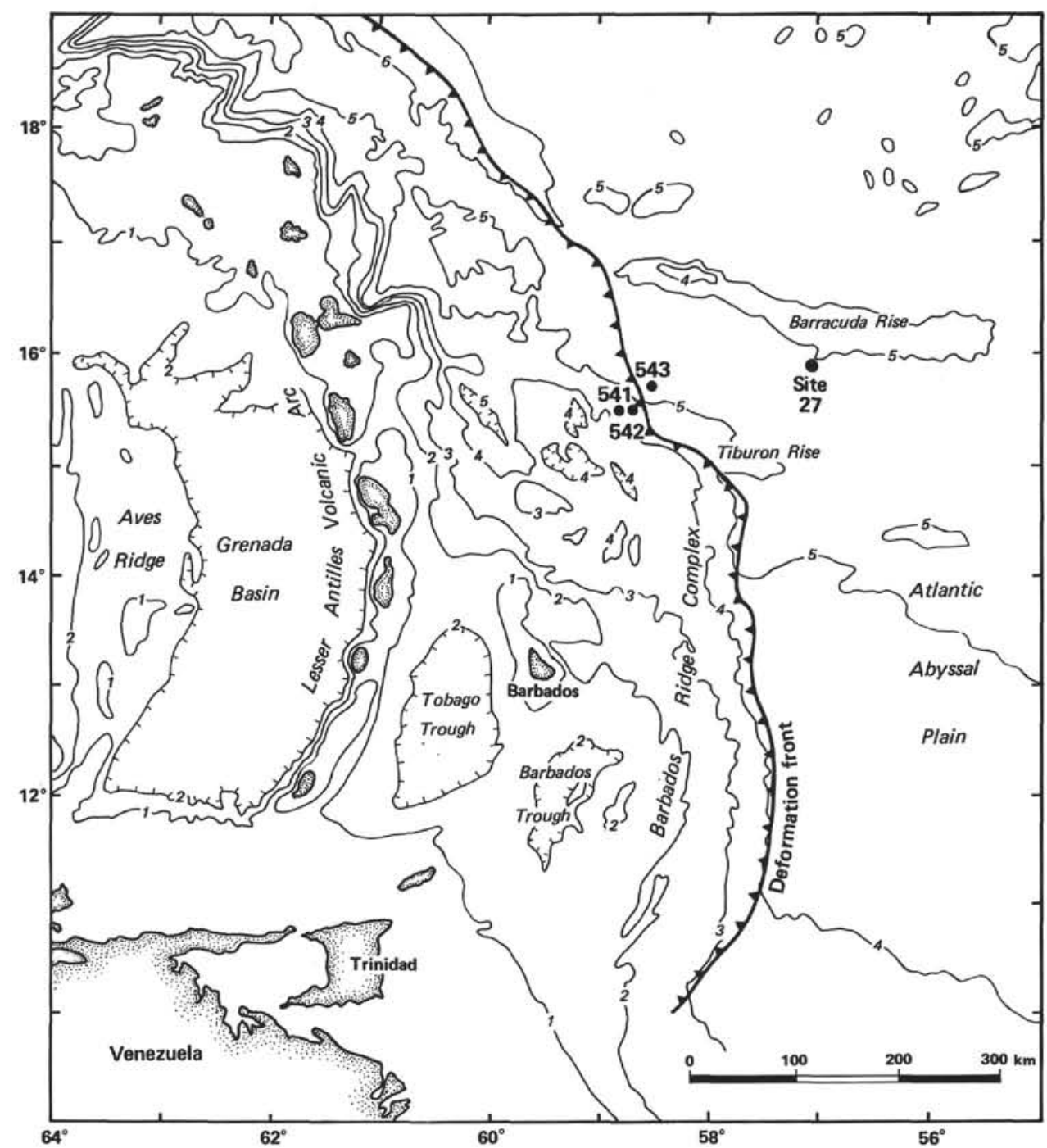

Figure 1. Locations of Sites 541, 542, and 543 on and near the Barbados Ridge.

Since the only reliable inclinations in the older samples are from the basalts, where secular variation has not been adequately averaged and tectonic tilting is probably significant, this problem has not been considered further

\section{POLARITY STRATIGRAPHY}

\section{Site 541}

The sedimentary section at site 541 (Hole 541) yielded by far the most success in terms of polarity stratigraphy, with good determinations for the interval from 59 to $200 \mathrm{~m}$ sub-bottom (Cores 8 to 22; see Fig. 2). (All depths hereafter are sub-bottom.) The first sample (B-1, $141 \mathrm{~cm} ; 59.4 \mathrm{~m}$ ) is normal, corresponding to either the oldest Brunhes $(0.7 \mathrm{Ma})$ or the Jaramillo event $(0.91-$ $0.97 \mathrm{Ma}$; ages are from Ness et al., 1980). Other recognizable markers are the Olduvai event $(1.66-1.87 \mathrm{Ma})$ at 88-90 m (Sections 11-2 and 11-3), the Matuyama/Gauss transition (2.47 Ma) at $120.5 \mathrm{~m}$ (Section 14-4), and the Gauss/Gilbert transition (3.40 Ma) at about $141 \mathrm{~m}$ (Section 16-6). The identification of the Olduvai event corresponds extremely well to the Pliocene/Pleistocene boundary at 90-91 m (Section 11-3), identified from both nan- nofossils and foraminifers (Site Report for Site 541, this volume). The first normal event in the Gilbert epoch (3.86 Ma) occurs at $156 \mathrm{~m}$ (Section 18-3), and if there is a fault at the base of Core $19(172 \mathrm{~m})$, as indicated by an inversion in the nannofossil stratigraphy (see Site Report), the polarity information would indicate about $20 \mathrm{~m}$ of repeated section, with the first normal event in the Gilbert occurring again at $178 \mathrm{~m}$ (Section 20-5). This correlation is consistent with the two thick ash beds in Core $18(18-1,140 \mathrm{~cm}[154.4 \mathrm{~m}]$ and $18-2,135 \mathrm{~cm}[155.8 \mathrm{~m}])$ being equivalent to the two thick beds in Core $20(20-1$, $119 \mathrm{~cm}[173.2 \mathrm{~m}]$ and $20-3,15 \mathrm{~cm}$ [175.2 m]). The polarity transition at $195 \mathrm{~m}$ (Section 22-3) probably corresponds to the last normal event in the Gilbert (4.79 Ma). It is very unfortunate that operational constraints prevented the use of the hydraulic piston corer, since the exceptionally high sediment accumulation rate, the reasonable magnetic stability, and the presence of datable ashes provide one of the best known opportunities to study polarity subchrons and excursions in the Brunhes and Matuyama polarity epochs.

From 289 to $340 \mathrm{~m}$ (Cores 32 to 37 ) a high percentage of the samples were horizontally magnetized, and the 
Table 1A. Sediment paleomagnetic data summary.

\begin{tabular}{|c|c|c|c|c|c|c|c|c|}
\hline & & & NRM & & & Optimu & m Demag & \\
\hline $\begin{array}{l}\text { Sample } \\
\text { (core-section, } \\
\mathrm{cm} \text { level) }\end{array}$ & $\begin{array}{l}\text { Sub-bottom } \\
\text { depth } \\
\text { (m) }\end{array}$ & $\begin{array}{c}\text { Incli- } \\
\text { nation } \\
\text { (degrees) }\end{array}$ & $\begin{array}{c}\text { Relative } \\
\text { decli- } \\
\text { nation } \\
\text { (degrees) }\end{array}$ & $\begin{array}{l}\text { Intensity } \\
\times 10^{-3} \\
(\mathrm{~A} / \mathrm{m})\end{array}$ & $\begin{array}{c}\text { Incli- } \\
\text { nation } \\
\text { (degrees) }\end{array}$ & $\begin{array}{l}\text { Relative } \\
\text { decli- } \\
\text { nation } \\
\text { (degrees) }\end{array}$ & $\begin{array}{l}\text { Intensity } \\
\times 10^{-3} \\
(\mathrm{~A} / \mathrm{m})\end{array}$ & $\begin{array}{c}\text { Demagne- } \\
\text { tization } \\
\text { level } \\
\text { (mT) }\end{array}$ \\
\hline Hole S41 & & & & & & & & \\
\hline $8-1,141$ & 59.41 & 47 & 232 & 3.0 & 33 & 222 & 1.0 & 20 \\
\hline $8-2,36$ & 59.86 & 6 & 312 & 1.7 & -20 & 244 & 3.6 & 16 \\
\hline $8-5,14$ & 64.14 & 8 & 331 & 7.9 & -3 & 330 & 4.9 & 24 \\
\hline $8-7,13$ & 67.13 & -12 & 55 & 5.8 & -14 & 59 & 4.7 & 16 \\
\hline $8-7,64$ & 67.64 & -12 & 73 & 4.8 & -22 & 63 & 4.3 & 10 \\
\hline $9-7,51$ & 77.01 & 11 & 182 & 4.0 & -7 & 112 & 3.2 & 20 \\
\hline $10-1,91$ & 77.91 & -26 & 41 & 2.4 & -32 & 31 & 3.6 & 10 \\
\hline $10-3,123$ & 81.23 & -3 & 147 & 10.0 & -13 & 148 & 8.5 & 10 \\
\hline $10-4,67$ & 82.17 & -34 & 350 & 2.3 & -63 & 323 & 1.3 & 10 \\
\hline $10-6,125$ & 85.75 & 2 & 22 & 14.8 & -6 & 32 & 9.4 & 20 \\
\hline $10-7,47$ & 86.47 & -43 & 30 & 0.6 & -20 & 116 & 0.8 & 20 \\
\hline $11-1,146$ & 87.96 & -8 & 188 & 9.7 & -11 & 191 & 5.0 & 16 \\
\hline $11-2,20$ & 88.20 & 28 & 133 & 3.4 & 33 & 134 & 1.7 & 13 \\
\hline $11-2,102$ & 89.02 & -5 & 32 & 6.3 & -5 & 35 & 4.0 & 16 \\
\hline $11-3,17$ & 89.67 & 17 & 96 & 14.4 & 31 & 107 & 7.7 & 16 \\
\hline $11-3,127$ & 90.77 & -3 & 300 & 3.7 & -2 & 297 & 2.3 & 13 \\
\hline $11-4,5$ & 91.05 & -12 & 126 & 2.1 & -26 & 100 & 1.6 & 13 \\
\hline $11-4,73$ & 91.73 & -8 & 224 & 6.0 & -8 & 224 & 6.0 & 13 \\
\hline $11-4,110$ & 92.10 & -6 & 233 & 6.9 & -16 & 229 & 6.1 & 13 \\
\hline $12-1,22$ & 96.22 & -54 & 272 & 4.3 & -59 & 259 & 3.0 & 13 \\
\hline $12-7,22$ & 105.22 & $?$ & 164 & 7.9 & -14 & 165 & 4.3 & 13 \\
\hline $12-7,70$ & 105.70 & 13 & 161 & 0.8 & -17 & 158 & 1.7 & 13 \\
\hline $13-1,77$ & 106.27 & -24 & 128 & 12.0 & -24 & 134 & 13.0 & 13 \\
\hline $13-3,103$ & 109.53 & -28 & 104 & 2.1 & -31 & 137 & 2.8 & 13 \\
\hline $13-4,113$ & 111.13 & -11 & 145 & 4.8 & -6 & 147 & 2.9 & 16 \\
\hline $13-5,65$ & 112.15 & 1 & 317 & 6.5 & 1 & 312 & 3.9 & 16 \\
\hline $13-5,135$ & 112.85 & 4 & 357 & 8.2 & 2 & 359 & 5.6 & 13 \\
\hline $13-6,89$ & 113.89 & -15 & 78 & 3.0 & -30 & 103 & 3.1 & 13 \\
\hline $13-7,60$ & 115.10 & -6 & 248 & 3.5 & -15 & 233 & 3.5 & 16 \\
\hline $14-1,40$ & 115.40 & -38 & 210 & 0.8 & -34 & 190 & 1.5 & 16 \\
\hline $14-1,116$ & 116.16 & 36 & 177 & 0.47 & 6 & 157 & 0.27 & 16 \\
\hline $14-2,61$ & 117.11 & -32 & 101 & 4.8 & -21 & 107 & 3.9 & 16 \\
\hline $14-2,132$ & 117.82 & -3 & 149 & 4.5 & 2 & 147 & 3.8 & 16 \\
\hline $14-3,150$ & 118.50 & -35 & 336 & 1.3 & -36 & 330 & 1.6 & 16 \\
\hline $14-3,121$ & 119.21 & 29 & 5 & 2.7 & 6 & 39 & 1.2 & 30 \\
\hline $14-4,10$ & 119.60 & -4 & 120 & 8.4 & -6 & 126 & 4.3 & 23 \\
\hline $14-4,70$ & 120.20 & -26 & 178 & 4.8 & -46 & 182 & 3.4 & 23 \\
\hline $14-4,141$ & 120.91 & 9 & 180 & 4.4 & 10 & 183 & 3.7 & 13 \\
\hline $14-5,54$ & 121.54 & 3 & 316 & 7.9 & -1 & 328 & 5.4 & 16 \\
\hline $14-6,56$ & 123.06 & 30 & 104 & 12.7 & 26 & 106 & 7.6 & 16 \\
\hline $14-6,120$ & 123.70 & 3 & 113 & 7.7 & 3 & 99 & 2.9 & 25 \\
\hline $14-7,60$ & 124.50 & 3 & 101 & 12.0 & -3 & 106 & 8.5 & 16 \\
\hline $15-1,105$ & 125.55 & 2 & 359 & 4.5 & -8 & 355 & 1.2 & 16 \\
\hline $15-2,55$ & 126.55 & 68 & 250 & 4.9 & 65 & 292 & 3.4 & 13 \\
\hline $15-3,52$ & 128.02 & 16 & 115 & 5.9 & 14 & 117 & 2.8 & 16 \\
\hline $15-4,55$ & 129.55 & 14 & 248 & 2.3 & 13 & 234 & 1.1 & 10 \\
\hline $15-5,16$ & 130.66 & 36 & 55 & 2.9 & 19 & 73 & 2.3 & 16 \\
\hline $15-5,90$ & 131.40 & 35 & 133 & 4.2 & 40 & 138 & 2.5 & 16 \\
\hline $15-6,16$ & 132.16 & 20 & 95 & 5.9 & 14 & 109 & 1.1 & 16 \\
\hline $16-2,58$ & 136.08 & 32 & 184 & 5.0 & -16 & 32 & 0.55 & 40 \\
\hline $16-2,138$ & 136.88 & 3 & 312 & 10.9 & -14 & 304 & 3.1 & 30 \\
\hline $16-3,58$ & 137.58 & 58 & 269 & 1.4 & -34 & 324 & 0.35 & 30 \\
\hline $16-4,31$ & 138.89 & 34 & 241 & 1.0 & -4 & 215 & 0.62 & 30 \\
\hline $16-4,100$ & 139.50 & 53 & 173 & 0.93 & 12 & 322 & 0.63 & 30 \\
\hline $16-5,47$ & 140.47 & 4 & 54 & 5.4 & 9 & 321 & 0.98 & 25 \\
\hline $16-6,88$ & 142.38 & -15 & 231 & 2.2 & -32 & 196 & 0.66 & 25 \\
\hline $17-1,42$ & 143.92 & -5 & 338 & 9.1 & -12 & 272 & 1.9 & 16 \\
\hline $17-2,128$ & 146.28 & 32 & 169 & 1.9 & -23 & 48 & 0.78 & 25 \\
\hline $17-3,28$ & 146.78 & 9 & 50 & 3.8 & -20 & 21 & 3.0 & 13 \\
\hline $17-3,102$ & 147.52 & -6 & 158 & 0.30 & -24 & 55 & 0.20 & 20 \\
\hline $17-4,74$ & 148.74 & 8 & 130 & 2.2 & -23 & 79 & 0.71 & 30 \\
\hline $18-1,39$ & 153.39 & 17 & 85 & 4.8 & 2 & 36 & 1.4 & 16 \\
\hline 50 d 20 & & 3 & 83 & & -17 & 36 & & \\
\hline $18-1,141$ & 154.41 & 13 & 255 & 14.4 & -34 & 281 & 4.3 & 30 \\
\hline $18-3,56$ & 156.56 & 56 & 299 & 1.5 & 50 & 338 & 0.62 & 45 \\
\hline$s 180$ d 20 & & 38 & 289 & & 40 & 320 & & \\
\hline $18-4,116$ & 158.56 & 23 & 94 & 5.9 & 70 & 49 & 0.95 & 50 \\
\hline$s 0 \mathrm{~d} 20$ & & 3 & 94 & & 51 & 67 & & \\
\hline $18-5,133$ & 160.33 & 60 & 125 & 5.1 & 48 & 183 & 1.7 & 23 \\
\hline s 0 d 20 & & 42 & 113 & & 45 & 162 & & \\
\hline $18-6,75$ & 161.25 & 10 & 108 & 4.7 & -10 & 120 & 0.35 & 26 \\
\hline $19-1,77$ & 163.27 & 20 & 332 & 2.1 & 4 & 358 & 1.4 & 16 \\
\hline s 130 d 40 & & 30 & 314 & & 32 & 348 & & \\
\hline $19-1,146$ & 163.96 & 43 & 116 & 0.2 & 6 & 303 & 0.14 & 25 \\
\hline $50 \mathrm{~d} 25$ & & & & & 27 & 308 & & \\
\hline $19-6,110$ & 171.10 & 10 & 193 & 35.7 & 13 & 213 & 11.6 & 25 \\
\hline$s 0 \mathrm{~d} 10$ & & 13 & 191 & & 18 & 210 & & \\
\hline $20-1,35$ & 172.35 & -52 & 92 & 0.25 & -10 & 40 & 0.80 & 40 \\
\hline $20-1,123$ & 173.23 & -2 & 22 & 2.1 & -28 & 326 & 0.48 & so \\
\hline $20-3,81$ & 175.81 & -8 & 22 & 0.57 & 0 & 329 & 0.80 & 30 \\
\hline $20-4,141$ & 177.91 & -14 & 327 & 3.5 & -13 & 329 & 1.7 & 20 \\
\hline $20-5,16$ & 178.16 & 23 & 263 & 28.7 & 20 & 300 & 2.9 & 40 \\
\hline $20-6,92$ & 180.42 & 44 & 43 & 2.9 & 60 & 5 & 1.7 & 30 \\
\hline $20-7,6$ & 181.06 & -12 & 89 & 1.4 & -12 & 94 & 1.1 & 10 \\
\hline $21-1,9$ & 181.59 & 29 & 99 & 2.8 & 44 & 22 & 1.2 & 20 \\
\hline $21-1,98$ & 182.48 & 7 & 166 & 6.5 & -14 & 137 & 3.5 & 20 \\
\hline $21-2,88$ & 183.88 & 27 & 268 & 15.0 & 3 & 300 & 5.7 & 25 \\
\hline
\end{tabular}


Table 1A. (Continued).

\begin{tabular}{|c|c|c|c|c|c|c|c|c|c|c|}
\hline \multirow[b]{2}{*}{$\begin{array}{c}\text { Sample } \\
\text { (core-section, } \\
\text { cm level) }\end{array}$} & \multirow[b]{2}{*}{$\begin{array}{l}\text { Sub-bottom } \\
\text { depth } \\
\text { (m) }\end{array}$} & & NRM & & & Optimun & m Demag & & & \\
\hline & & $\begin{array}{c}\begin{array}{c}\text { Incli- } \\
\text { nation } \\
\text { (degrees) }\end{array} \\
\end{array}$ & $\begin{array}{c}\begin{array}{c}\text { Relative } \\
\text { decli- } \\
\text { nation } \\
\text { (degrees) }\end{array} \\
\end{array}$ & $\begin{array}{l}\text { Intensity } \\
\times 10^{-3} \\
(\mathrm{~A} / \mathrm{m})\end{array}$ & $\begin{array}{c}\begin{array}{c}\text { Incli- } \\
\text { nation } \\
\text { (degrees) }\end{array} \\
\text { (d) }\end{array}$ & $\begin{array}{c}\begin{array}{c}\text { Relative } \\
\text { decli- } \\
\text { nation } \\
\text { (degrees) }\end{array} \\
\end{array}$ & $\begin{array}{l}\text { Intensity } \\
\times 10^{-3} \\
(\mathrm{~A} / \mathrm{m})\end{array}$ & $\begin{array}{c}\text { Demagne- } \\
\text { tization } \\
\text { level } \\
(\mathrm{mT})\end{array}$ & Polarity & $\begin{array}{l}\text { Relia- } \\
\text { bilitya }\end{array}$ \\
\hline Hole 541 (Cont.) & & & & & & & & & & \\
\hline $21-3,25$ & 184.75 & 4 & 92 & 3.6 & -25 & 5 & 2.1 & 20 & $\mathbf{R}$ & A \\
\hline $21-3,102$ & 185.52 & 11 & 104 & 10.5 & 16 & 22 & 1.4 & 35 & N & $c+$ \\
\hline $\begin{array}{l}22-1,64 \\
=5150 \mathrm{~d} 25\end{array}$ & 191.64 & 17 & 292 & 4.1 & $\begin{array}{l}20 \\
17\end{array}$ & $\begin{array}{l}329 \\
320\end{array}$ & 0.70 & 35 & $\mathrm{~N}$ & A \\
\hline $22-1,128$ & 192.28 & 23 & 153 & 4.8 & 14 & 145 & 0.49 & 40 & $\mathrm{~N}$ & A- \\
\hline $22-2,62$ & 193.12 & 31 & 230 & 8.3 & 32 & 289 & 1.9 & 30 & $\mathrm{~N}$ & A \\
\hline $22-3,58$ & 194.58 & 24 & 92 & 5.2 & 13 & 341 & 1.9 & 25 & $\mathrm{~N}$ & A \\
\hline $22-4,7$ & 195.57 & -16 & 260 & 50.5 & -28 & 254 & 23.5 & 35 & R & A \\
\hline $22-4,128$ & 196.78 & 7 & 84 & 3.7 & -34 & 351 & 1.6 & 30 & $\hat{\mathbf{R}}$ & A \\
\hline $22-5,60$ & 197.60 & -44 & 116 & 0.80 & 4 & 358 & 0.26 & 30 & $?$ & $\mathrm{~A} / \mathrm{H}$ \\
\hline $22-6,42$ & 198.92 & 0 & 83 & 0.88 & -20 & 21 & 0.48 & 25 & $\mathbf{R}$ & A \\
\hline $22-6,130$ & 199.80 & -2 & 18 & 15.9 & -32 & 325 & 7.4 & 25 & R & A \\
\hline $23-1,78$ & 201.28 & -15 & 87 & 4.6 & 20 & 46 & 1.8 & 16 & $\mathrm{n}$ & $\mathrm{n}$ \\
\hline $8310 \mathrm{~d} 15$ & & 4 & 85 & & 5 & 46 & 1.0 & 10 & $?$ & $\mathrm{~A} / \mathrm{H}$ \\
\hline $23-5,35$ & 207.28 & 8 & 316 & 7.1 & -16 & 336 & 0.81 & 60 & ${ }^{\circ}$ & 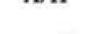 \\
\hline $\mathrm{s} 150 \mathrm{~d} 40$ & & 0 & 312 & & -8 & 345 & & & R? & $\mathrm{A}-/ \mathrm{H}$ \\
\hline 23-6, 94 & 208.94 & 1 & 5 & 39.5 & 3 & 5 & 19.2 & 20 & $?$ & $\mathbf{A} / \mathbf{H}$ \\
\hline $32-3,29$ & 289.29 & 5 & 53 & 6.2 & 14 & 314 & 0.95 & 23 & & \\
\hline s $210 \mathrm{~d} 45$ & & 20 & 43 & & -30 & 315 & & & $\mathbf{R}$ & A- \\
\hline $33-5,11$ & 301.61 & 41 & 267 & 22.6 & 4 & 149 & 8.2 & 23 & $?$ & $\mathrm{~A} / \mathrm{H}$ \\
\hline $33-6$, is & 303.15 & 35 & 204 & 9.8 & 7 & 154 & 0.60 & 20 & & \\
\hline 50 d 20 & & 41 & 189 & & -2 & 153 & & & $?$ & $\mathrm{C} / \mathrm{H}$ \\
\hline $33-6,75$ & 303.75 & 39 & 177 & 4.6 & 42 & 161 & 0.95 & 13 & & \\
\hline $\begin{array}{l}5.50 \text { d } 45 \\
33-7,45\end{array}$ & 304.95 & $\begin{array}{l}0 \\
8\end{array}$ & 168 & 03 & -4 & $\begin{array}{l}157 \\
217\end{array}$ & & 35 & $?$ & $\mathrm{~A} / \mathrm{H}$ \\
\hline $\begin{array}{l}33-7,45 \\
\text { s } 150 \text { d } 25\end{array}$ & & $\begin{array}{r}8 \\
12\end{array}$ & $\begin{array}{l}137 \\
142\end{array}$ & 0.3 & $\begin{array}{l}53 \\
29\end{array}$ & $\begin{array}{l}217 \\
224\end{array}$ & 0.13 & 30 & $\mathrm{~N}$ & A- \\
\hline $34-1,32$ & 305.32 & 57 & 309 & 20.3 & 3 & 77 & 19.1 & 26 & & \\
\hline $560 \mathrm{~d} 30$ & & 79 & 248 & & -6 & 76 & & & ? & $\mathrm{A} / \mathrm{H}$ \\
\hline $34-5,67$ & 311.67 & 36 & 243 & 75.9 & 21 & 190 & 6.0 & 30 & & \\
\hline $590 \mathrm{~d} 20$ & & 25 & 233 & & 2 & 189 & & & $?$ & $\mathrm{C} / \mathrm{H}$ \\
\hline $34-6,46$ & 312.96 & 36 & 310 & 45.7 & 67 & 223 & 6.4 & 30 & & $c$ \\
\hline $\begin{array}{c}5180 \mathrm{~d} 30 \\
35-5,66\end{array}$ & 321.16 & 12 & 302 & & 42 & 248 & 15 & 26 & $\mathrm{~N}$ & C \\
\hline $540 \mathrm{~d} 45$ & 321.16 & $\begin{array}{c}32 \\
-7\end{array}$ & $\begin{array}{l}164 \\
159\end{array}$ & 7.7 & $\begin{array}{r}0 \\
-39\end{array}$ & $\begin{array}{r}104 \\
95\end{array}$ & 1.5 & 20 & $\mathbf{R}$ & c \\
\hline $37-2,109$ & 336.09 & 4 & 86 & 0.64 & -7 & 333 & 0.10 & 25 & & \\
\hline S $200 \mathrm{~d} 20$ & & 22 & 84 & & -21 & 336 & & & $\mathrm{R}$ ? & D \\
\hline $37-3,81$ & 337.31 & 18 & 121 & 1.0 & -12 & 67 & 0.9 & 30 & & \\
\hline $8330 \mathrm{~d} 25$ & & 5 & 117 & & -37 & 69 & & & $\mathbf{R}$ & A \\
\hline $37-5,78$ & 340.28 & 18 & 183 & 11.1 & 18 & 193 & 4.9 & 30 & & \\
\hline sod 15 & & 18 & 178 & & 21 & 188 & & & $\mathrm{~N}$ & A \\
\hline $41-4,57$ & 376.57 & 23 & 122 & 9.2 & 10 & 60 & 2.6 & 16 & N? & c \\
\hline $41-4,107$ & 377.07 & 36 & 146 & 14.5 & 16 & 91 & 5.2 & 16 & $\mathrm{~N}$ & c \\
\hline $42-1,103$ & 382.03 & 36 & 276 & 43.5 & 35 & 283 & 26.1 & 16 & $\mathrm{~N}$ & A \\
\hline $42-2,98$ & 383.48 & 36 & 214 & 47.8 & 37 & 220 & 23.3 & 16 & $\mathbf{N}$ & A \\
\hline $42-3,106$ & 385.06 & 36 & 105 & 18.8 & -9 & 20 & 3.2 & 30 & R? & c \\
\hline $42-4,64$ & 386.14 & 23 & 65 & 27.2 & 23 & 68 & 13.3 & 16 & $\mathrm{~N}$ & B \\
\hline $42-5,3$ & 387.03 & 33 & 358 & 29.3 & 34 & 322 & 8.5 & 16 & $\mathrm{~N}$ & B \\
\hline $43-5,69$ & 397.19 & 41 & 328 & 66.0 & 31 & 327 & 27.0 & 10 & & \\
\hline 5120 d 125 & & -5 & 252 & & 1 & 259 & & & ? & $\mathrm{A} / \mathrm{H}$ \\
\hline $43-5,77$ & 397.27 & 24 & 108 & 59.3 & 20 & 113 & 41.0 & 10 & & \\
\hline $\begin{array}{l}\text { s? ? d } 125 \\
43-6,109\end{array}$ & 399.09 & 27 & 222 & 63.6 & 25 & & 28.6 & & $\stackrel{?}{\mathrm{~N}}$ & $\underset{B}{A}$ \\
\hline 444,148 & 405.98 & 32 & 67 & $\begin{array}{l}18.9 \\
18.9\end{array}$ & $\begin{array}{r}25 \\
-36\end{array}$ & $\begin{array}{l}228 \\
331\end{array}$ & $\begin{array}{r}28.6 \\
3.7\end{array}$ & $\begin{array}{l}16 \\
45\end{array}$ & $\begin{array}{l}\mathbf{N} \\
\mathbf{R}\end{array}$ & $\begin{array}{l}\mathrm{B} \\
\mathrm{C}\end{array}$ \\
\hline $44-5,78$ & 406.78 & 28 & 40 & 118.8 & 24 & 40 & 86.5 & 10 & N & B \\
\hline $44-6,82$ & 408.32 & 32 & 174 & 47.1 & 38 & 142 & 7.6 & 25 & $\mathrm{~N}$ & c \\
\hline $44-7,55$ & 409.55 & 40 & 136 & 86.2 & 32 & 132 & 57.4 & 10 & $\mathrm{~N}$ & B \\
\hline $45-1,141$ & 410.91 & 39 & 358 & 19.2 & 32 & 2 & 13.4 & 10 & $\mathrm{~N}$ & B \\
\hline $45-2,27$ & 411.27 & 27 & 297 & 98.7 & 31 & 304 & 64.6 & 10 & N & B \\
\hline $45-3,61$ & 413.11 & 38 & 62 & $\begin{array}{l}68.7 \\
68.1\end{array}$ & 38 & 57 & 46.4 & 10 & $\mathrm{~N}$ & B \\
\hline $45-7,17$ & 418.67 & 37 & 255 & 18.3 & 36 & 259 & 13.5 & 10 & $\mathrm{~N}$ & B \\
\hline $48-4,10$ & 442.60 & 2 & 260 & 9.7 & -6 & 284 & 0.3 & 16 & ? & C \\
\hline $48-4,108$ & 443.58 & & 41 & 12.3 & 50 & 44 & 7.2 & 10 & $\mathrm{~N}$ & B \\
\hline $48-6,65$ & 446.15 & 12 & 349 & 25.5 & 0 & 336 & 10.0 & 16 & $?$ & c \\
\hline $48-6,102$ & 446.52 & -21 & 232 & 18.6 & -15 & 230 & 11.7 & 10 & $\mathbf{R}$ & B \\
\hline $48, \mathrm{CC}(15)$ & 447.65 & 11 & 135 & 7.5 & -56 & 258 & 0.6 & 16 & $? ?$ & $\mathbf{F}$ \\
\hline $49-2,115$ & 450.15 & 15 & 66 & 17.6 & 6 & 85 & 1.8 & 13 & $?$ & $\mathrm{~A} / \mathrm{H}$ \\
\hline $49-2,129$ & 450.29 & & & & -6 & 227 & 3.4 & 16 & & \\
\hline $49-3,42$ & 450.92 & 23 & 80 & 1.2 & 13 & 93 & 0.5 & 13 & $\mathrm{~N}$ ? & c \\
\hline $49-4,32$ & 452.32 & 33 & 167 & 18.1 & 25 & 149 & 9.6 & 16 & $\mathrm{~N}$ & C \\
\hline $49-4,138$ & 453.38 & 16 & 302 & 20.5 & 6 & 286 & 9.0 & 16 & $?$ & c \\
\hline $49-5,52$ & 454.02 & 66 & 333 & 8.6 & & & & & & \\
\hline Hole 542 & & & & & & & & & & \\
\hline H2-2, 108 & & -19 & 186 & 0.91 & -7 & 237 & 0.40 & 5 & ? & c \\
\hline $\mathrm{H} 2-7,98$ & & 4 & 124 & 12.5 & 0 & 120 & 5.9 & 16 & ? & $\mathrm{A} / \mathrm{H}$ \\
\hline H3-1, 145 & & -13 & 117 & 48.3 & -11 & 108 & 30.7 & 16 & & \\
\hline s $300 \mathrm{~d} 25$ & & -13 & 123 & & -14 & 114 & & & $\mathbf{R}$ & A \\
\hline $\mathrm{H} 3-3,97$ & & -11 & 133 & 4.1 & -8 & 143 & 0.96 & 10 & $\mathbf{R}$ ? & A \\
\hline $1-1,14$ & 202.14 & 23 & 337 & 12.7 & 17 & 344 & 2.1 & 10 & $\mathrm{~N}$ & B \\
\hline $1-2,99$ & 204.49 & -18 & 109 & 18.8 & -10 & 97 & 18.9 & 16 & $\mathbf{R}$ & A \\
\hline $1-3,52$ & 205.52 & -36 & 135 & 46.1 & -36 & 131 & 32.9 & 10 & $\mathbf{R}$ & B \\
\hline $1-4,35$ & 206.85 & -13 & 281 & 39.0 & -15 & 263 & 18.8 & 16 & $\ddot{R}$ & A \\
\hline $1-4,103$ & 207.53 & 23 & 49 & 0.77 & -9 & 316 & 0.18 & 50 & & \\
\hline s 330 d 12 & & & & & -6 & 314 & & & $?$ & $\mathrm{C} / \mathrm{H}$ \\
\hline $\begin{array}{l}1-6,69 \\
2-2,119\end{array}$ & $\begin{array}{l}210.19 \\
214.19\end{array}$ & $\begin{array}{r}11 \\
-36\end{array}$ & $\begin{array}{r}76 \\
337\end{array}$ & $\begin{array}{l}25.1 \\
12.4\end{array}$ & $\begin{array}{r}4 \\
22\end{array}$ & $\begin{array}{l}89 \\
59\end{array}$ & $\begin{array}{l}5.4 \\
4.2\end{array}$ & $\begin{array}{l}16 \\
16\end{array}$ & $\stackrel{?}{N}$ & $\mathrm{~A} / \mathrm{H}$ \\
\hline $2-2,119$ & & & 337 & 12.4 & 22 & 59 & 4.2 & & & \\
\hline
\end{tabular}


Table 1A. (Continued).

\begin{tabular}{|c|c|c|c|c|c|c|c|c|c|c|}
\hline \multirow[b]{2}{*}{$\begin{array}{c}\text { Sample } \\
\text { (core-section, } \\
\mathrm{cm} \text { level) }\end{array}$} & \multirow[b]{2}{*}{$\begin{array}{l}\text { Sub-bottom } \\
\text { depth } \\
\text { (m) }\end{array}$} & & NRM & & & Optimun & n Demag & & & \\
\hline & & $\begin{array}{c}\text { Incli- } \\
\text { nation } \\
\text { (degrees) }\end{array}$ & $\begin{array}{l}\text { Relative } \\
\text { decli- } \\
\text { nation } \\
\text { (degrees) }\end{array}$ & $\begin{array}{l}\text { Intensity } \\
\times 10^{-3} \\
(\mathrm{~A} / \mathrm{m})\end{array}$ & $\begin{array}{c}\begin{array}{c}\text { Incli- } \\
\text { nation } \\
\text { (degrees) }\end{array} \\
\end{array}$ & $\begin{array}{l}\text { Relative } \\
\text { decli- } \\
\text { nation } \\
\text { (degrees) }\end{array}$ & $\begin{array}{l}\text { Intensity } \\
\times 10^{-3} \\
(\mathrm{~A} / \mathrm{m})\end{array}$ & $\begin{array}{l}\text { Demagne- } \\
\text { tization } \\
\text { level } \\
(\mathrm{mT}) \\
\end{array}$ & Polarity & $\begin{array}{l}\text { Relia- } \\
\text { bilitya }\end{array}$ \\
\hline Hole 542 (Cont.) & & & & & & & & & & \\
\hline $2-3,50$ & 215.00 & -54 & 94 & 0.74 & -46 & 94 & 0.40 & 5 & $\mathbf{R}$ ? & c \\
\hline $2-5,100$ & 218.50 & 14 & 44 & 11.0 & 10 & 59 & 1.2 & 16 & $\mathbf{N}$ & A \\
\hline $\begin{array}{l}3-2,95 \\
50 \mathrm{~d} 10\end{array}$ & 223.95 & -40 & 176 & 12.0 & $\begin{array}{l}-28 \\
-29\end{array}$ & $\begin{array}{l}185 \\
190\end{array}$ & 7.7 & 16 & $\mathbf{R}$ & A \\
\hline $3-3,67$ & 224.67 & -20 & 93 & 2.2 & -21 & 101 & 1.8 & 10 & 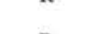 & . \\
\hline s $30 \mathrm{~d} 10$ & & -28 & 91 & & -30 & 100 & & & $\mathbf{R}$ & A \\
\hline $3-5,120$ & 228.20 & $\begin{array}{l}-43 \\
-55\end{array}$ & 136 & 0.95 & -38 & $\begin{array}{l}329 \\
319\end{array}$ & 0.09 & 15 & $\mathbf{R}$ & c \\
\hline $\begin{array}{l}\begin{array}{rl}5 & 0 \text { de } 20 \\
3-6,38\end{array}\end{array}$ & 228.88 & $\begin{array}{l}-55 \\
-35\end{array}$ & $\begin{array}{l}155 \\
110\end{array}$ & 1.7 & $\begin{array}{r}-26 \\
12\end{array}$ & $\begin{array}{l}319 \\
348\end{array}$ & 0.05 & 40 & $\pi$ & 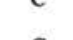 \\
\hline s 180 d 10 & 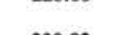 & -25 & 108 & & 9 & 347 & & & ? & c \\
\hline $4-1,42$ & 230.92 & -22 & 99 & 2.6 & -12 & 292 & 0.27 & 25 & & \\
\hline s $150 \mathrm{~d} 30$ & & 1 & 96 & & -29 & 302 & & & $\mathbf{R}$ & c \\
\hline $4-5,55$ & 237.05 & 8 & 134 & 8.8 & 19 & 124 & 2.3 & 13 & & \\
\hline s 180 d 18 & & 20 & 130 & & 33 & 130 & & & $\mathrm{~N}$ & A \\
\hline Hole 542A & & & & & & & & & & \\
\hline $2-4,132$ & 245.82 & 16 & 306 & 18.4 & 19 & 302 & 12.6 & 16 & & \\
\hline s $300 \mathrm{~d} 25$ & & 12 & 312 & & 16 & 310 & & & $\mathbf{N}$ & A+ \\
\hline $2-6,76$ & 248.26 & -62 & 80 & 12.0 & -54 & 138 & 14.0 & 20 & 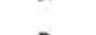 & . \\
\hline s $300 \mathrm{~d} 35$ & & -64 & 156 & & -34 & 167 & & & $\mathbf{R}$ & A+ \\
\hline $3-2,25$ & 251.25 & 25 & 331 & 15.7 & 30 & 330 & 11.8 & 16 & & \\
\hline s $340 \mathrm{~d} 25$ & & 27 & 341 & & 32 & 342 & & & $\mathbf{N}$ & $\mathrm{A}+$ \\
\hline $3-6,72$ & 257.72 & ${ }_{24}^{6}$ & 269 & 10.2 & $7^{7}$ & 267 & 3.8 & 13 & & \\
\hline $50 \mathrm{~d} 18$ & & 24 & 269 & & 24 & 266 & & & N & $\mathrm{A}+$ \\
\hline $4-1,32$ & 259.32 & -26 & 162 & 26.3 & -26 & 156 & 26.6 & 16 & $\mathbf{R}$ & A \\
\hline $4-1,82$ & 259.82 & 20 & 295 & 20.3 & 36 & 287 & 2.6 & 16 & $\mathrm{~N}$ & c \\
\hline $4-4,108$ & 264.58 & -49 & 347 & 2.5 & -38 & 197 & 2.7 & 20 & 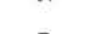 & 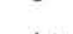 \\
\hline$s 40 \mathrm{~d} 15$ & & -36 & 340 & & -42 & 209 & & & $\mathbf{R}$ & A+ \\
\hline Hole 543 & & & & & & & & & & \\
\hline $6-6,142$ & 57.42 & -38 & 40 & 3.8 & -45 & 34 & 2.4 & 16 & $\mathbf{R}$ & A \\
\hline $7-4,8$ & 62.58 & -11 & 115 & 9.1 & -20 & 99 & 5.6 & 16 & $\mathbf{R}$ & A \\
\hline $8-5,123$ & 74.73 & -11 & 173 & 4.7 & -12 & 166 & 6.0 & 16 & $\mathbf{R}$ & A \\
\hline $9-3,22$ & 80.22 & 15 & 110 & 2.3 & -9 & 151 & 1.4 & 16 & $\mathbf{R}$ ? & A \\
\hline $10-5,33$ & 92.83 & 24 & 36 & 11.6 & 15 & 42 & 5.2 & 16 & $\mathrm{~N}$ & A \\
\hline $11-3,133$ & 100.33 & 24 & 12 & 4.5 & 7 & 12 & 3.3 & 25 & $?$ & c \\
\hline $12-1,8$ & 105.58 & -23 & 351 & 4.6 & -29 & 310 & 2.0 & 13 & $\mathbf{R}$ & c \\
\hline $12-1,48$ & 105.98 & -53 & 250 & 4.6 & -28 & 271 & 5.1 & 16 & $\mathbf{R}$ & A \\
\hline $13-2,19$ & 116.69 & 11 & 278 & 7.1 & 12 & 277 & 5.1 & 16 & $\mathrm{~N}$ & A \\
\hline $13-3,131$ & 119.31 & 40 & 105 & 0.5 & 15 & 27 & 5.8 & 20 & $\mathrm{~N}$ & c \\
\hline $13-5,125$ & 122.25 & 26 & 105 & 2.4 & 14 & 10 & 3.4 & 20 & $\mathrm{~N}$ ? & c \\
\hline $14-1,7$ & 124.57 & 4 & 45 & 11.4 & -5 & 16 & 6.3 & 30 & $?$ & c \\
\hline $14-2,139$ & 127.39 & 12 & 291 & 1.6 & 9 & 333 & 5.2 & 25 & ? & c \\
\hline $15-2,5$ & 135.55 & -70 & 203 & 3.5 & 3 & 323 & 3.8 & 30 & $?$ & c \\
\hline $17-1,138$ & 154.38 & 35 & 80 & 8.2 & 34 & 95 & 1.8 & 16 & $\mathrm{~N}$ & A \\
\hline $17-2,99$ & 155.49 & 4 & 53 & 1.6 & -20 & 23 & 2.5 & 16 & $\mathbf{R}$ & A \\
\hline $17-3,32$ & 156.32 & 10 & 352 & 8.7 & 0 & 336 & 1.7 & 20 & $?$ & $\mathrm{~A} / \mathrm{H}$ \\
\hline $17-4,16$ & 157.66 & 27 & 306 & 8.9 & 22 & 312 & 1.5 & 16 & $\mathrm{~N}$ & A \\
\hline $17-4,141$ & 158.91 & 60 & 331 & 2.8 & 39 & 331 & 1.1 & 20 & $\mathrm{~N}$ & c \\
\hline $18-1,35$ & 162.85 & 34 & 318 & 10.6 & 21 & 319 & 2.3 & 16 & $\mathrm{~N}$ & A \\
\hline $18-2,113$ & 165.13 & 26 & 346 & 0.6 & 40 & 12 & 0.5 & 13 & $\mathbf{N}$ & C \\
\hline $18-3,126$ & 166.76 & 47 & 224 & 10.2 & 40 & 208 & 3.2 & 16 & $\mathrm{~N}$ & A \\
\hline $18-4,53$ & 167.53 & 64 & 67 & 3.5 & -18 & 184 & 0.9 & 25 & $\mathbf{R}$ & c \\
\hline $18-6,93$ & 170.93 & -8 & 116 & 1.7 & -25 & 113 & 4.8 & 20 & $\mathbf{R}$ & A \\
\hline $19-1,78$ & 172.78 & 38 & 323 & 3.8 & -13 & 216 & 0.7 & 20 & R? & c \\
\hline $19-3,26$ & 175.26 & 8 & 349 & 3.5 & 10 & 334 & 1.0 & 16 & $\mathrm{~N}$ & A \\
\hline $19-4,80$ & 177.30 & 61 & 196 & 5.7 & 44 & 219 & 1.0 & 13 & N & A \\
\hline $19-5,92$ & 178.92 & 42 & 89 & 2.9 & 36 & 115 & 0.34 & 20 & $\mathrm{~N}$ & A \\
\hline $19-7,3$ & 181.03 & -5 & 273 & 1.4 & -22 & 244 & 0.52 & 13 & $\mathbf{R}$ & A \\
\hline $20-1,89$ & 182.39 & -14 & 218 & 1.9 & -39 & 206 & 3.4 & 16 & R & c \\
\hline $20-2,52$ & 183.52 & -44 & 42 & 0.9 & -70 & 19 & 0.57 & 13 & $\mathbf{R}$ & C \\
\hline $20-3,27$ & 184.77 & 36 & 65 & 10.5 & 19 & 69 & 1.9 & 16 & $\mathrm{~N}$ & A \\
\hline $20-3,113$ & 185.63 & 31 & 35 & 1.4 & -28 & 358 & 1.7 & 16 & $R$ & c \\
\hline $20-4,18$ & 186.18 & -1 & 358 & 2.4 & -32 & 258 & 1.9 & 16 & $\mathbf{R}$ & A \\
\hline $20-4,122$ & 187.22 & 37 & 323 & 8.1 & 6 & 337 & 1.1 & 25 & $?$ & $\mathrm{C} / \mathrm{H}$ \\
\hline $23-2,139$ & 212.89 & 15 & 118 & 1.0 & -5 & 153 & 0.79 & 20 & ? & $\mathrm{C} / \mathrm{H}$ \\
\hline $23-3,23$ & 213.23 & 33 & 250 & 1.6 & 26 & 243 & 0.72 & 16 & $\mathrm{~N}$ & $\mathrm{~A}$ \\
\hline $24-2,144$ & 222.44 & -66 & 321 & 0.42 & -71 & 340 & 0.40 & 13 & R & A- \\
\hline $24-4,18$ & 224.18 & -31 & 312 & 0.55 & -67 & 4 & 0.30 & 13 & R & c \\
\hline $24-4,98$ & 224.98 & -71 & 245 & 0.24 & -13 & 24 & 0.08 & 20 & R? & c \\
\hline $25-1,48$ & 229.48 & -68 & 352 & 0.32 & -59 & 325 & 0.36 & 13 & $\mathbf{R}$ & c \\
\hline $25-2,49$ & 230.99 & -18 & 28 & 1.6 & -30 & 294 & 0.25 & 16 & R & c \\
\hline $25-2,115$ & 231.65 & 9 & 339 & 3.1 & -38 & 246 & 0.87 & 16 & $\mathbf{R}$ & A \\
\hline $25-3,29$ & 232.29 & 25 & 330 & 1.6 & -19 & 195 & 0.78 & 16 & $\mathbf{R}$ & c \\
\hline $26-2,46$ & 240.46 & 56 & 356 & 2.9 & 1 & 335 & 2.3 & 20 & $?$ & $\mathrm{C} / \mathrm{H}$ \\
\hline $26-2,114$ & 241.14 & 49 & 263 & 4.2 & -34 & 103 & 1.4 & 16 & R & A \\
\hline $26-3,35$ & 241.85 & -15 & 319 & 2.7 & 7 & 285 & 0.43 & 25 & $?$ & $\mathrm{~A} / \mathrm{H}$ \\
\hline $26-3,85$ & 242.35 & 37 & 190 & 3.3 & -34 & 98 & 0.57 & 16 & R & C \\
\hline $26-4,43$ & 243.43 & 46 & 181 & 3.1 & -19 & 13 & 0.74 & 13 & $\mathbf{R}$ & A \\
\hline $26-4,103$ & 244.03 & 20 & 18 & 4.0 & -4 & 349 & 0.16 & 25 & $?$ & C- \\
\hline $26-5,73$ & 245.23 & 62 & 104 & 3.6 & -24 & 312 & 1.4 & 16 & $R$ & A \\
\hline $26-6,44$ & 246.44 & 28 & 13 & 2.1 & -3 & 198 & 0.57 & 20 & $?$ & $\mathrm{~A} / \mathrm{H}$ \\
\hline $26-6,121$ & 247.21 & 73 & 77 & 2.1 & -34 & 353 & 1.1 & 13 & R & C \\
\hline $27-2,121$ & 250.71 & 31 & 297 & 3.8 & -9 & 126 & 2.4 & 16 & $\mathbf{R}$ & A \\
\hline $27-3,39$ & 251.39 & 38 & 344 & 3.2 & -73 & 215 & 0.43 & 16 & $\vec{R}$ & A \\
\hline
\end{tabular}


Table 1A. (Continued).

\begin{tabular}{|c|c|c|c|c|c|c|c|c|c|c|}
\hline \multirow[b]{2}{*}{$\begin{array}{c}\text { Sample } \\
\text { (core-section, } \\
\text { cm level) }\end{array}$} & \multirow[b]{2}{*}{$\begin{array}{l}\text { Sub-bottom } \\
\text { depth } \\
\text { (m) }\end{array}$} & \multicolumn{3}{|c|}{ NRM } & \multicolumn{4}{|c|}{ Optimum Demag } & \multirow[b]{2}{*}{ Polarity } & \multirow[b]{2}{*}{$\begin{array}{l}\text { Relia- } \\
\text { bilitya }\end{array}$} \\
\hline & & $\begin{array}{c}\text { Incli- } \\
\text { nation } \\
\text { (degrees) }\end{array}$ & $\begin{array}{l}\text { Relative } \\
\text { decli- } \\
\text { nation } \\
\text { (degrees) }\end{array}$ & $\begin{array}{l}\text { Intensity } \\
\times 10^{-3} \\
(\mathrm{~A} / \mathrm{m})\end{array}$ & $\begin{array}{c}\text { Incli- } \\
\text { nation } \\
\text { (degrees) }\end{array}$ & $\begin{array}{l}\text { Relative } \\
\text { decli- } \\
\text { nation } \\
\text { (degrees) }\end{array}$ & $\begin{array}{l}\text { Intensity } \\
\times 10^{-3} \\
(\mathrm{~A} / \mathrm{m})\end{array}$ & $\begin{array}{l}\text { Demagne- } \\
\text { tization } \\
\text { level } \\
\text { (mT) }\end{array}$ & & \\
\hline \multicolumn{11}{|l|}{ Hole 543 (Cont.) } \\
\hline $27-4,113$ & 253.63 & 69 & 79 & 4.6 & -21 & 300 & 1.4 & 16 & $\mathbf{R}$ & A \\
\hline $27-5,4$ & 254.04 & 66 & 60 & 4.2 & $\begin{array}{l}-21 \\
-15\end{array}$ & 292 & 2.1 & 16 & $\hat{R}$ & A \\
\hline $28-2,27$ & 259.27 & 30 & 191 & 10.6 & -11 & 190 & 1.8 & 35 & $\hat{\mathbf{R}}$ & $\mathrm{c}$ \\
\hline $28-3,27$ & 260.77 & 43 & 40 & 7.5 & 22 & 84 & 0.54 & 20 & $\mathrm{~N}$ & A \\
\hline $28-3,131$ & 261.81 & 19 & 307 & 6.6 & -26 & 313 & 0.30 & 16 & $\mathbf{R}$ & c \\
\hline $28-4,38$ & 262.38 & 26 & 19 & 7.9 & -40 & 350 & 0.2 & 16 & R? & D \\
\hline $28-4,94$ & 262.94 & 19 & 333 & 7.8 & -33 & 182 & 0.16 & 16 & R? & D \\
\hline $28-5,54$ & 264.04 & 32 & 236 & 7.2 & -1 & 217 & 1.6 & 25 & $?$ & A/H \\
\hline $29-1,111$ & 268.11 & 18 & 336 & 7.8 & -14 & 327 & 1.8 & 20 & R & $\mathrm{c}$ \\
\hline $29-2,111$ & 269.61 & 66 & 204 & 4.6 & -10 & 10 & 1.7 & 20 & $\hat{\mathbf{R}}$ & A \\
\hline $29-3,24$ & 270.24 & 44 & 343 & 5.2 & $\begin{array}{l}-10 \\
-35\end{array}$ & 183 & 0.55 & 16 & $\hat{\mathbf{R}}$ & c \\
\hline $29-3,111$ & 271.11 & 27 & 24 & 5.1 & $\begin{array}{l}-16 \\
-16\end{array}$ & 203 & 0.54 & 13 & $\hat{R}$ & c \\
\hline $29-4,111$ & 272.61 & 30 & 340 & 3.6 & -17 & 242 & 0.41 & 20 & $\hat{\mathbf{R}}$ & $\mathrm{c}+$ \\
\hline $29-5,111$ & 274.11 & 80 & 56 & 2.3 & $\begin{array}{l}-11 \\
-10\end{array}$ & 253 & $\begin{array}{l}3.41 \\
3.3\end{array}$ & 16 & $\hat{R}$ & $\mathrm{C}$ \\
\hline $29-6,24$ & 274.74 & 35 & 3 & 1.6 & 7 & 308 & 0.17 & 30 & ? & c \\
\hline $29-6,111$ & 275.61 & 36 & 168 & 6.8 & -7 & 172 & 1.3 & 20 & $R$ ? & C \\
\hline $29-7,20$ & 276.20 & 1 & 91 & 4.4 & 5 & 102 & 1.2 & 20 & $?$ & A/H \\
\hline $30-1,99$ & 277.49 & 18 & 34 & 10.0 & -4 & 36 & 1.4 & 20 & $?$ & $\mathrm{~A} / \mathrm{H}$ \\
\hline $30-2,94$ & 278.94 & 29 & 334 & 5.4 & -83 & 325 & 0.32 & 16 & $\mathrm{R}$ ? & D \\
\hline $30-3,93$ & 280.43 & 19 & 26 & 5.6 & -5 & 73 & 1.0 & 20 & $?$ & $\mathrm{~A} / \mathrm{H}$ \\
\hline $30-4,105$ & 282.05 & -10 & 31 & 3.9 & -5 & 231 & 1.3 & 20 & $?$ & $\mathrm{~A} / \mathrm{H}$ \\
\hline $30-5,107$ & 283.57 & 60 & 14 & 4.2 & -14 & 346 & 0.95 & 25 & $\mathbf{R}$ & A \\
\hline $30-6,56$ & 284.56 & 85 & 310 & 4.8 & $\begin{array}{l}13 \\
-13\end{array}$ & 292 & 2.3 & 16 & $\hat{\mathbf{R}}$ & A \\
\hline $31-1,32$ & 286.32 & 27 & 264 & 6.7 & -11 & 245 & 1.1 & 16 & $\begin{array}{l}\hat{R} \\
\mathbf{R}\end{array}$ & A \\
\hline $31-2,14$ & 287.64 & 25 & 53 & 5.9 & -15 & 58 & 0.82 & 20 & $\ddot{R}$ & c \\
\hline $32-1,91$ & 296.41 & 42 & 248 & 1.2 & 11 & 63 & 0.46 & 16 & $\mathrm{~N}$ ? & c \\
\hline $32-2,103$ & 298.03 & -11 & 285 & 1.3 & -18 & 282 & 1.7 & 16 & $\mathbf{R}$ & A \\
\hline $32-3,92$ & 299.42 & 5 & 280 & 0.79 & -13 & 245 & 0.74 & 16 & $\hat{R}$ & c \\
\hline $32-4,122$ & 301.22 & -6 & 115 & 1.0 & -20 & 121 & 1.4 & 16 & R & A \\
\hline $32-5,89$ & 302.39 & -59 & 351 & 0.62 & -71 & 52 & 0.82 & 13 & $\hat{\mathbf{R}}$ & A- \\
\hline $33-1,94$ & 305.94 & 14 & $\begin{array}{l}181 \\
181\end{array}$ & 2.5 & 1 & 187 & $\begin{array}{l}1.02 \\
1.4\end{array}$ & 25 & ? & $\mathrm{A} / \mathrm{H}$ \\
\hline $33-2,70$ & 307.20 & $\begin{array}{r}14 \\
8\end{array}$ & $\begin{array}{l}101 \\
266\end{array}$ & 2.6 & -10 & $\begin{array}{l}167 \\
267\end{array}$ & $\begin{array}{l}1.4 \\
1.5\end{array}$ & 25 & R & $\mathrm{C}$ \\
\hline $34-1,102$ & 315.52 & -25 & 133 & 1.5 & -25 & 128 & 1.4 & 16 & R & A + \\
\hline $34-2,23$ & 316.23 & -61 & 266 & 1.1 & -59 & 271 & 1.2 & 16 & $\hat{R}$ & At \\
\hline \multicolumn{11}{|l|}{ Hole 543A } \\
\hline $3-1,3$ & 341.53 & 21 & 218 & 4.6 & 6 & 206 & 2.0 & 20 & $?$ & $\mathrm{~A}+/ \mathrm{H}$ \\
\hline $3-1,40$ & 341.90 & 32 & $\begin{array}{r}69 \\
69\end{array}$ & 3.6 & 9 & 152 & 1.1 & 25 & N? & $\mathrm{A}+/ \mathrm{H}$ \\
\hline $5-1,104$ & 361.54 & -26 & 337 & $\begin{array}{l}3.0 \\
4.3\end{array}$ & -58 & 166 & 1.1 & 25 & R & A \\
\hline $5-2,81$ & 362.81 & $\begin{array}{r}-20 \\
5\end{array}$ & 326 & $\begin{array}{l}5.3 \\
5.3\end{array}$ & $\begin{array}{l}-58 \\
-9\end{array}$ & $\begin{array}{l}100 \\
326\end{array}$ & 0.28 & 25 & $\begin{array}{l}\mathrm{K} \\
\mathrm{R} \text { ? }\end{array}$ & $\mathrm{C}+/ \mathrm{H}$ \\
\hline $5-3,94$ & 364.44 & 11 & 16 & 11.2 & 17 & 39 & 1.3 & 25 & $\hat{N}$ & $\mathrm{~A}+$ \\
\hline $6-1,18$ & 370.18 & 25 & 303 & 3.9 & -4 & 311 & 0.47 & 40 & $?$ & $\mathrm{C} / \mathrm{H}$ \\
\hline $7-2,103$ & 382.03 & 20 & 342 & 11.7 & 35 & 262 & 0.46 & 20 & $\mathrm{~N}$ & A- \\
\hline $7-3.7$ & 382.57 & 48 & 290 & 0.39 & 31 & 157 & 0.09 & 35 & $\mathrm{~N}$ & C \\
\hline $7-3,14$ & 382.64 & -5 & 358 & 12.3 & -23 & 335 & 1.2 & 25 & R & A \\
\hline $7-3,85$ & 383.35 & 17 & 6 & 18.6 & 39 & 17 & 1.5 & 25 & $\mathrm{~N}$ & A- \\
\hline $8-1,76$ & 389.76 & -15 & 33 & 11.0 & -31 & 354 & 2.5 & 20 & R & A \\
\hline $9-1,46$ & 398.96 & 4 & 351 & 23.1 & $\begin{array}{l}-11 \\
-48\end{array}$ & 33 & 0.63 & 20 & ? & $\mathrm{F}$ \\
\hline $10-1,11$ & 408.11 & 52 & 75 & 5.3 & 24 & 156 & 3.3 & 25 & $\mathrm{~N}$ & A \\
\hline
\end{tabular}

Note: For samples from dipping beds, a second line of data gives strike (s), dip (d), and corrected inclinations and declinations.

${ }^{2}$ Reliability categories: $\mathrm{A}=$ stable, multiple demagnetizations; $\mathrm{B}=$ stable, single demagnetization; $\mathrm{C}=$ direction changing systemati cally at highest demagnetization level; $\mathrm{D}=$ marginally stable; $\mathrm{F}=$ unstable; $\mathrm{H}=$ too close to horizontal for polarity determination. (See text.)

few confident polarity determinations were too far apart for polarity stratigraphy. The samples from 382 to $419 \mathrm{~m}$ (Cores 42 to 44 ) at first looked promising. Fourteen of 15 samples have inclinations between $21^{\circ}$ and $38^{\circ}$ (without tectonic correction), and all of these samples have very high intensities and show exceptional directional stability. However, applying the tectonic correction to the two samples from the overturned section in Section 43-5 results in nearly horizontal magnetizations, strongly suggesting a post-deformational magnetization. This interpretation is supported by the failure to observe similar properties of intensity or stability in any samples that might be the same age at Site 543 . The most likely mechanism for resetting the magnetization is chemical or thermal changes associated with the warm water observed at the bottom of the hole.

\section{Site 542}

The number of sediment specimens available from Site 542 was inadequate for polarity stratigraphy, even though the samples were quite stable and horizontal magnetizations were rare. In Hole 542, the predominantly reversed interval from 200 to $230 \mathrm{~m}$ (Cores 1 to 4 ) probably corresponds to the Gilbert epoch, but this assignment is based more on the nannofossil age than on the polarity intervals.

\section{Site 543}

At Site 543, drilling deformation prevented adequate sampling of the sediments in the upper $150 \mathrm{~m}$ (Hole 543, Cores 1 to 16). Sampling was adequate in Cores 17 to 20 $(150-190 \mathrm{~m})$, and a correlation can be drawn with Anomalies 5C through 5E (16 to $19 \mathrm{Ma}$ ) of the reversal time scale (Fig. 3). This is in agreement with the radiolarian age assignments of $D$. alata to $S$. wolffii (see Site Report for Site 543, this volume) based on the time scale of Theyer and Hammond (1974). Cores 21 and 22 had no recovery, and Cores 23 through 34 (the bottom of Hole 543) show a dramatic change in directional properties. The vast majority of the samples have reversed sta- 
Table 1B. Basalt paleomagnetic data, Hole 543A.

\begin{tabular}{|c|c|c|c|c|c|c|c|c|c|}
\hline \multirow[b]{2}{*}{$\begin{array}{c}\text { Sample } \\
\text { (core-section, } \\
\mathrm{cm} \text { level) }\end{array}$} & \multirow[b]{2}{*}{$\begin{array}{l}\text { Sub-bottom } \\
\text { depth } \\
\text { (m) }\end{array}$} & \multicolumn{3}{|c|}{ NRM } & \multicolumn{4}{|c|}{ Optimum Demag } & \multirow[b]{2}{*}{$\begin{array}{l}\text { Susceptibility } \\
\left(10^{-4} \mathrm{cgs}\right)\end{array}$} \\
\hline & & $\begin{array}{c}\text { Incli- } \\
\text { nation } \\
\text { (degrees) }\end{array}$ & $\begin{array}{l}\text { Relative } \\
\text { decli- } \\
\text { nation } \\
\text { (degrees) }\end{array}$ & $\begin{array}{l}\text { Intensity } \\
(\mathrm{A} / \mathrm{m})\end{array}$ & $\begin{array}{c}\text { Incli- } \\
\text { nation } \\
\text { (degrees) }\end{array}$ & $\begin{array}{l}\text { Relative } \\
\text { decli- } \\
\text { nation } \\
\text { (degrees) }\end{array}$ & $\begin{array}{c}\text { Intensity } \\
(\mathrm{A} / \mathrm{m})\end{array}$ & $\begin{array}{c}\text { Demagne- } \\
\text { tization } \\
\text { level } \\
(\mathrm{mT})\end{array}$ & \\
\hline $10-1,127$ & 409.27 & -11.1 & 163.1 & 2.71 & -11.8 & 161.4 & 1.82 & 20 & 3.1 \\
\hline $10-2,32$ & 409.82 & -29.5 & 268.7 & 5.74 & -29.4 & 270.2 & 3.95 & 20 & 7.6 \\
\hline $11-1,40$ & 417.90 & -36.9 & 359.1 & 5.16 & -35.9 & 0.1 & 3.71 & $450^{\circ} \mathrm{Ca}$ & 6.1 \\
\hline $11-1,140$ & 418.90 & -34.9 & 272.5 & 8.56 & -32.5 & 274.1 & 5.05 & 20 & 7.2 \\
\hline $11-2,85$ & 419.85 & -28.8 & 198.2 & 5.16 & -30.8 & 198.6 & 2.28 & 20 & 9.1 \\
\hline $12-1,32$ & 420.32 & -31.9 & 210.6 & 8.25 & -31.8 & 211.6 & 4.74 & $450^{\circ} \mathrm{C}$ & 6.8 \\
\hline $12-2,6$ & 421.56 & -20.5 & 139.1 & 7.56 & -20.9 & 135.6 & 4.45 & 20 & 10.0 \\
\hline $12-2,64$ & 422.14 & -19.3 & 202.9 & 6.76 & -19.8 & 204.2 & 3.09 & 20 & 8.3 \\
\hline $12-3,40$ & 423.40 & -21.9 & 107.1 & 4.06 & -23.7 & 107.8 & 2.82 & 20 & \\
\hline $12-4,135$ & 425.85 & -21.6 & 132.0 & 5.33 & -20.9 & 130.9 & 3.66 & 20 & 6.1 \\
\hline $13-1,26$ & 427.26 & -21.4 & 88.6 & 6.70 & -21.0 & 88.4 & 5.74 & 20 & 3.2 \\
\hline $13-2,93$ & 429.43 & -22.1 & 211.9 & 5.41 & & & & & \\
\hline $13-3,74$ & 430.74 & -32.7 & 234.2 & 5.03 & -31.4 & 238.3 & 1.37 & 20 & 12.3 \\
\hline $13-5,40$ & 433.40 & -27.9 & 4.0 & 9.67 & -27.8 & 7.5 & 6.42 & $340^{\circ} \mathrm{C}$ & 6.8 \\
\hline $13-6,114$ & 435.64 & -23.7 & 276.5 & 6.12 & -24.0 & 276.4 & 2.14 & 20 & 10.7 \\
\hline $13-7,42$ & 436.42 & -26.2 & 172.0 & 6.10 & -24.4 & 168.3 & 0.89 & 20 & 16.8 \\
\hline $14-1,28$ & 436.28 & -23.6 & 90.3 & 6.94 & -19.2 & 86.2 & 1.29 & 20 & 12.8 \\
\hline $15-1,35$ & 438.35 & -25.4 & 335.3 & 14.5 & -26.4 & 335.8 & 10.9 & $340^{\circ} \mathrm{C}$ & 6.0 \\
\hline $15-2,14$ & 439.64 & -28.8 & 250.3 & 5.56 & -27.1 & 255.6 & 1.25 & 20 & 13.7 \\
\hline $15-3,70$ & 441.70 & -22.5 & 196.4 & 10.6 & -21.2 & 197.4 & 2.95 & 20 & 11.2 \\
\hline $15-4,117$ & 443.67 & -27.7 & 224.2 & 8.35 & -28.3 & 229.9 & 2.38 & 20 & 10.4 \\
\hline $15-5,65$ & 444.65 & -23.2 & 177.7 & 7.95 & -23.2 & 177.2 & 2.72 & 20 & 9.2 \\
\hline $16-1,94$ & 445.94 & -28.0 & 232.4 & 8.98 & -27.7 & 235.1 & 3.99 & 20 & 8.5 \\
\hline $16-2,38$ & 446.88 & -20.7 & 96.2 & 9.47 & -19.3 & 94.4 & 4.11 & 20 & 7.8 \\
\hline $16-3,127$ & 449.27 & -21.7 & 180.5 & 9.01 & -20.8 & 180.5 & 2.39 & 20 & 10.6 \\
\hline $16-4,91$ & 450.41 & -21.5 & 145.8 & 9.23 & -19.6 & 144.3 & 2.52 & 20 & 9.7 \\
\hline $16-5,78$ & 451.78 & -17.9 & 191.1 & 10.5 & -19.4 & 191.5 & 2.53 & 20 & 11.2 \\
\hline $16-6,88$ & 453.38 & -21.1 & 14.1 & 10.9 & -22.8 & 14.1 & 5.18 & $340^{\circ} \mathrm{C}$ & 5.1 \\
\hline $16-7,86$ & 454.86 & -32.9 & 272.4 & 5.70 & -31.5 & 273.6 & 3.16 & 20 & 6.6 \\
\hline
\end{tabular}

Note: See Table $1 \mathrm{~A}$ footnotes for reliability catetories and other information.

a Temperature of thermal demagnetization.

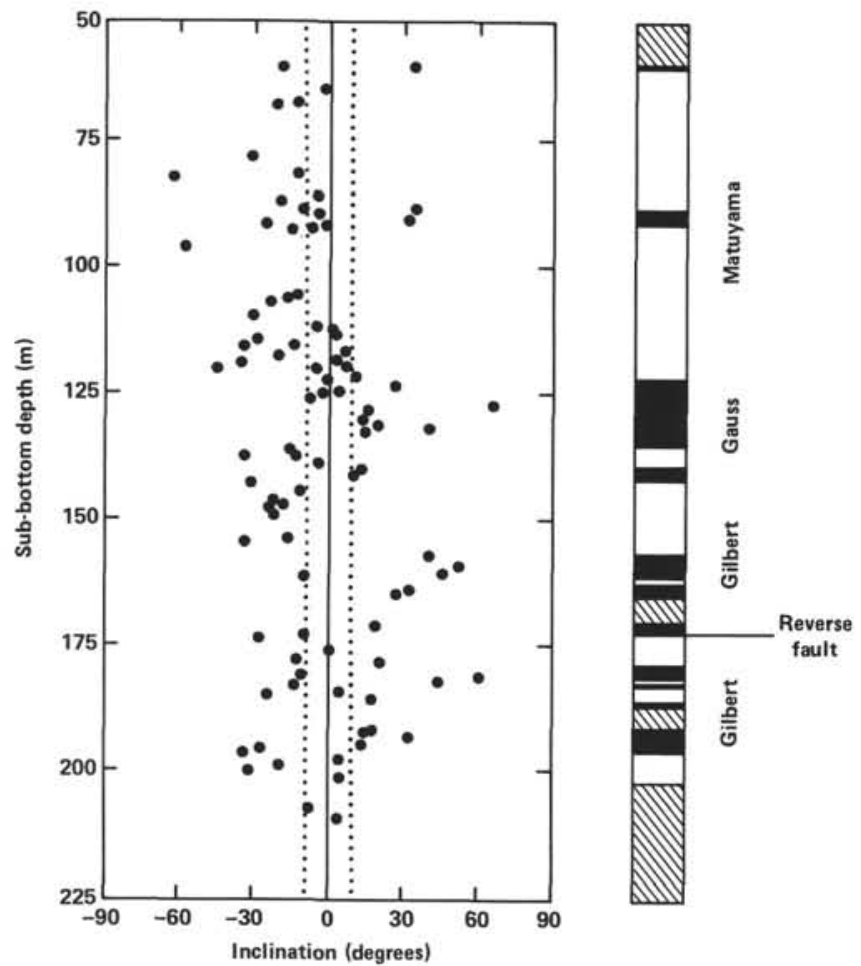

Figure 2. Inclinations and polarity interpretations for Site 541 . The dotted lines indicate the $10^{\circ}$ cutoff for polarity determinations. Black indicates normal polarity; white, reversed; and hachured, indeterminate.

ble directions, though most have normal NRM's. Since the age range of these samples covers most of the Eocene and Oligocene, some type of post-depositional re- magnetization is clearly indicated. A likely cause of the remagnetization is the diagenetic changes associated with the formation of manganese staining and of authigenic rhodochrosite. There is very little information as to the age of this remagnetization.

The Paleocene(?) and Upper Cretaceous sediments of Hole 543A were only sparsely sampled, owing to drilling deformation. Polarities are mixed; about a third of the samples have nearly horizontal magnetization. The age of magnetization of these iron-rich sediments is open to question, however, since reddish staining fronts were observed to crosscut bedding features.

\section{STRUCTURAL INTERPRETATION}

The interpretation of in situ structure provided interesting results. The objective is to use the magnetic declination of samples taken at dipping beds to estimate the original orientation and thereby determine approximately the true dip directions of the beds. Of the samples collected at dipping beds for Site 541, twelve have very stable directions, and eight of these have unambiguous polarity. For Site 542, nine samples at apparently dipping beds have very stable magnetizations. However, Hole 542 was abandoned because it was measured to deviate $7.6^{\circ}$ from vertical, so three samples with only moderate dips from this hole have been excluded. For the samples with clearly determined polarity, true north was assumed to be the declination direction for normal samples, and declination minus $180^{\circ}$ for reversed rocks. The apparent in situ strikes and down-dip directions in Table 2 and Figure 4 are relative to this north. For any one sample, the uncertainty in this rather crude technique is estimated to be about $40^{\circ}$. The main sources of error are the lack of 


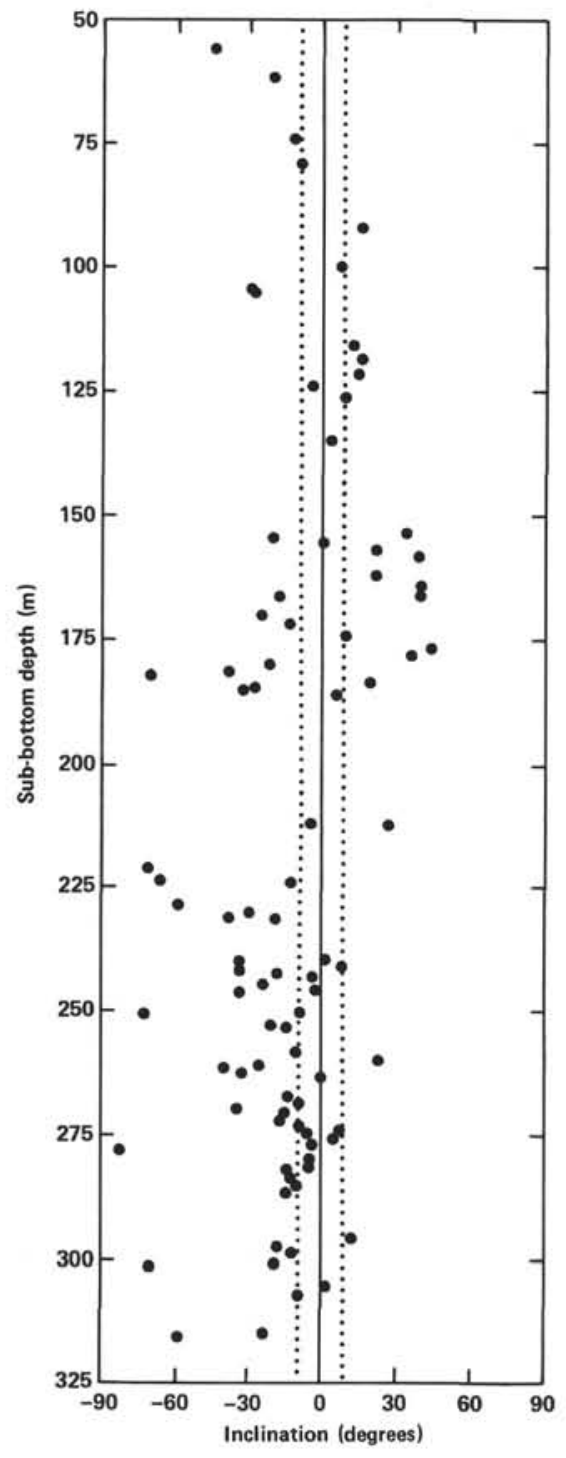

Figure 3. Inclinations and polarity interpretations for Site 543. Symbols as in Figure 2 .

correspondence between the declination direction and true north, and the uncertainty in measuring the orientation of a slightly deformed bed on half of a 10-cm-diameter core. From Site 541, the eight samples with clear polarity scatter about dip directions to the southwest, and the samples with ambiguous polarity are not inconsistent with this. For Site 542, located about $1.5 \mathrm{~km}$ to the east, the six samples dip predominantly to the east. The scatter within each site is remarkably small, considering the uncertainties. These dipping beds, often with dips of $30^{\circ}$ to $45^{\circ}$, alternate with horizontal beds over depth ranges of typically 5 to $10 \mathrm{~m}$. The in situ pattern suggested by horizontal beds alternating with dipping beds with the same down-dip direction is that the regions of horizontal bedding are separated by bands of monoclinal folds, with the axial plane of the folds probably dipping in the opposite direction from the bedding dip. These fold bands may well be controlled by subparallel thrust faults.
Table 2. Structural interpretation.

\begin{tabular}{|c|c|c|c|c|c|c|}
\hline \multirow[b]{2}{*}{$\begin{array}{c}\text { Sample } \\
\text { (core-section, } \\
\mathrm{cm} \text { level) }\end{array}$} & \multicolumn{2}{|c|}{$\begin{array}{c}\text { Bedding } \\
\text { (relative to core) }\end{array}$} & \multicolumn{2}{|c|}{$\begin{array}{l}\text { Magnetization } \\
\text { (corrected } \\
\text { for bedding) }\end{array}$} & \multirow[b]{2}{*}{ Polarity } & \multirow{2}{*}{$\begin{array}{c}\text { Apparent } \\
\text { in situ } \\
\text { down-dip } \\
\text { direction } \\
\text { (degrees) }\end{array}$} \\
\hline & $\begin{array}{c}\text { Strike } \\
\text { (degrees) }\end{array}$ & $\begin{array}{c}\text { Dip } \\
\text { (degrees) }\end{array}$ & $\begin{array}{c}\text { Incli- } \\
\text { nation } \\
\text { (degrees) }\end{array}$ & $\begin{array}{c}\text { Decli- } \\
\text { nation } \\
\text { (degrees) }\end{array}$ & & \\
\hline \multicolumn{7}{|l|}{ Hole 541} \\
\hline $\begin{array}{l}18-1,39 \\
18-5,133 \\
19-1,77 \\
19-6,110 \\
22-1,64 \\
32-3,29 \\
37-3,81 \\
37-5,78\end{array}$ & $\begin{array}{r}300 \\
0 \\
130 \\
0 \\
150 \\
210 \\
330 \\
0\end{array}$ & $\begin{array}{l}20 \\
20 \\
40 \\
10 \\
25 \\
45 \\
25 \\
15\end{array}$ & $\begin{array}{r}-17 \\
45 \\
32 \\
18 \\
17 \\
-30 \\
-37 \\
21\end{array}$ & $\begin{array}{r}36 \\
162 \\
348 \\
210 \\
320 \\
315 \\
69 \\
188\end{array}$ & $\begin{array}{l}\mathrm{R} \\
\mathrm{N} \\
\mathrm{N} \\
\mathrm{N} \\
\mathrm{N} \\
\mathrm{R} \\
\mathrm{R} \\
\mathrm{N}\end{array}$ & $\begin{array}{l}174 \\
288 \\
232 \\
240 \\
280 \\
165 \\
171 \\
262\end{array}$ \\
\hline \multicolumn{7}{|c|}{ Polarity ambiguous } \\
\hline $\begin{array}{l}23-1,78 \\
23-5,78 \\
33-6,75 \\
34-1,32\end{array}$ & $\begin{array}{r}310 \\
150 \\
50 \\
60\end{array}$ & $\begin{array}{l}15 \\
40 \\
45 \\
30\end{array}$ & $\begin{array}{r}5 \\
4 \\
-4 \\
-6\end{array}$ & $\begin{array}{r}46 \\
317 \\
157 \\
76\end{array}$ & & $\begin{array}{r}174 \text { or } 354 \\
103 \text { or } 283 \\
103 \text { or } 283 \\
74 \text { or } 254\end{array}$ \\
\hline \multicolumn{7}{|l|}{ Hole 542} \\
\hline H3-1, 145 & 300 & 25 & -14 & 114 & $\mathbf{R}$ & 96 \\
\hline \multicolumn{7}{|l|}{ Hole 542A } \\
\hline $\begin{array}{l}2-4,132 \\
2-6,76 \\
3-2,25 \\
3-6,72 \\
4-4,108\end{array}$ & $\begin{array}{r}300 \\
300 \\
340 \\
0 \\
40\end{array}$ & $\begin{array}{l}25 \\
35 \\
25 \\
18 \\
15\end{array}$ & $\begin{array}{r}16 \\
-34 \\
32 \\
24 \\
-42\end{array}$ & $\begin{array}{l}310 \\
167 \\
342 \\
266 \\
209\end{array}$ & $\begin{array}{l}\mathbf{N} \\
\mathbf{R} \\
\mathbf{N} \\
\mathbf{N} \\
\mathbf{R}\end{array}$ & $\begin{array}{r}80 \\
43 \\
88 \\
184 \\
101\end{array}$ \\
\hline
\end{tabular}

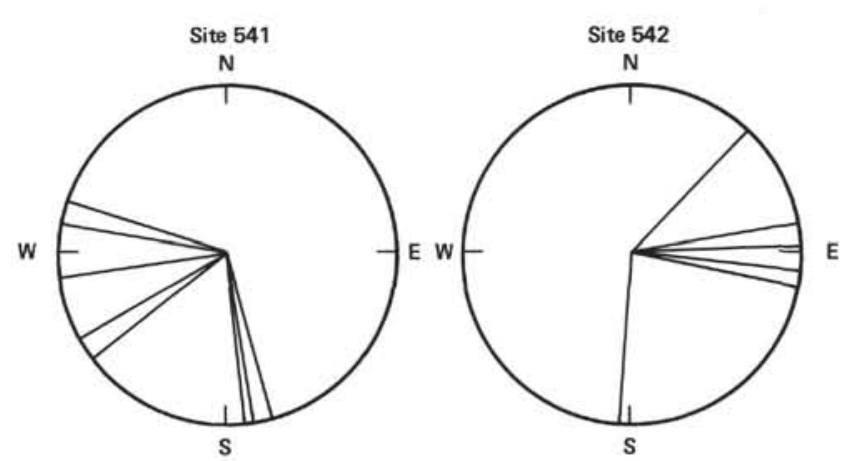

Figure 4. Estimates of in situ down-dip direction, based on the difference between magnetic declination and bedding strike relative to the core. Each radial line represents the down-dip direction for a single bed.

\section{BASALT MAGNETIZATION}

Coring of Hole 543A recovered $35.9 \mathrm{~m}$ of basalt at an average recovery rate of $81 \%$. The lithology was moderately altered plagioclase-olivine phyric pillow basalt. These flows are dated as Campanian (approx $80 \mathrm{Ma}$ old) on the basis of overlying and interbedded sediment, and compositionally are typical of normal ocean crust (e.g., Bougault et al., this volume). The freedom from limitation of the sample interval and the high degree of magnetic stability provided a pleasant contrast to the sedimentary section. The sample interval averaged about $1.5 \mathrm{~m}$, and the remanence direction rarely moved more than $3^{\circ}$ to $4^{\circ}$ during either AF or thermal demagnetization. There is no indication of drilling-induced, viscous, or other type of unstable magnetization. The samples from Hole $543 \mathrm{~A}$ can be divided into three groups ac- 
cording to their inclinations: group 1 consists of a single sample from Section 10-1 (409 m), with an inclination of $-11^{\circ}$; group 2 includes Sections 10-2 to 12-1 (410$420 \mathrm{~m}$ ), with inclinations of $-29^{\circ}$ to $-36^{\circ}$; and group 3 includes Sections $12-2$ to $16-7(421-455 \mathrm{~m})$, with inclinations of $-19^{\circ}$ to $-31^{\circ}$. These groupings are very similar to those based on petrography (Natland et al., this volume). The interpretation of these distinct mineralogic and magnetic groups is that they represent brief eruptive events separated by fairly long time intervals.

The mean NRM intensity of the basalt samples is 7.45 $\mathrm{A} / \mathrm{m}$, with a standard deviation of 2.50 . Using the results from Sites 417 and 418 (Levi et al., 1979) as a standard for Cretaceous basalts, these intensities fall well within the normal scatter. The mean susceptibility is $8.8 \times 10^{-4}$ cgs units, with standard deviation $3.1 \times 10^{-4}$. This is again within the normal range, perhaps below average. The Königsberger ratio $Q$ of remanent to induced magnetization scatters between 10 and 50 ; most values are near 20. The median destructive fields determined from AF demagnetizations range from io to $25 \mathrm{mT}$, again, these values are not unusual.

\section{SUMMARY}

Magnetostratigraphy for Leg 78A met with limited success. For Site 541, the interval from 60 to $200 \mathrm{~m}$ was correlated with the Matuyama through Gilbert polarity epochs. For Site 543, the interval from 150 to $190 \mathrm{~m}$ was correlated with marine magnetic Anomalies 5C through 5E. Down-dip directions of tilted beds inferred from declination values for Sites 541 and 542 suggest a pattern of monoclinal folding. Results from basalts are comparable to those for other DSDP sites.

\section{ACKNOWLEDGMENTS}

Reviews by Shaul Levi and Fritz Theyer improved the manuscript.

\section{REFERENCES}

Levi, S., Bleil, U., Smith, B. M., and Rigotti, P. A., 1979. Compilation of paleomagnetic and rock magnetic results of basalt samples from DSDP Legs 51, 52, and 53. In Donnelly, T., Francheteau, J., Bryan, W., Robinson, P., Flower, M., Salisbury, M., et al., Init. Repts. DSDP, 51, 52, 53, Pt. 2: Washington (U.S. Govt. Printing Office), $1337-1350$.

Ness, G., Levi, S., and Couch, R., 1980. Marine magnetic anomaly timescales for the Cenozoic and Late Cretaceous: a précis, critique, and synthesis. Rev. Geophys. Space Phys., 18:753-770.

Theyer, F., and Hammond, S. R., 1974. Paleomagnetic polarity sequence and radiolarian zones, Brunhes to polarity epoch 20 . Earth Planet. Sci. Lett., 22:307-319.

Date of Initial Receipt: July 25, 1983

Date of Acceptance: August 19, 1983 